\title{
Gender differences in the relationships among neurosteroid serum levels, cognitive function, and quality of life
}

This article was published in the following Dove Press journal:

Neuropsychiatric Disease and Treatment

\author{
Chien-Yu Chen' \\ Chih-Ching $\mathrm{Wu}^{2,3}$ \\ Yu-Chi Huang' \\ Chi-Fa Hung' \\ Liang-Jen Wang ${ }^{4}$
}

'Department of Psychiatry, Kaohsiung Chang Gung Memorial Hospital, Chang Gung University College of Medicine, Kaohsiung, Taiwan; ${ }^{2}$ Molecular Medicine Research Center, Chang Gung University, Tao-Yuan, Taiwan; ${ }^{3}$ Department of Medical Biotechnology and Laboratory Science, Chang Gung University, Tao-Yuan, Taiwan; ${ }^{4}$ Department of Child and Adolescent Psychiatry, Kaohsiung Chang Gung Memorial Hospital, Chang Gung University College of Medicine, Kaohsiung, Taiwan
Correspondence: Liang-Jen Wang Department of Child and Adolescent Psychiatry, Kaohsiung Chang Gung Memorial Hospital, No I23,

Ta-Pei Road, Kaohsiung City, Taiwan

Tel +886773 I 7123 ext 8753

Fax +88677326817

Email wangliangjen@gmail.com
Background: Dehydroepiandrosterone (DHEA), its sulfate ester (DHEA-S), and pregnenolone are neurosteroids that can be synthesized in the brain. Previous studies have hypothesized that these neurosteroids have antiaging, mood-enhancing, and cognitive-preserving effects; however, these effects may be gender-specific. Therefore, the purpose of this study was to investigate the gender differences in the relationships among neurosteroids (DHEA, DHEA-S, and pregnenolone), cognitive function, and quality of life in healthy individuals.

Method: In this cross-sectional study, we enrolled 47 men (mean age: 32.8 years) and 75 women (mean age: 35.4 years) who had no major physical or psychiatric illnesses and measured their serum DHEA, DHEA-S, and pregnenolone. Furthermore, we evaluated the subjects' cognitive function and quality of life using the Brief Assessment of Cognition in Schizophrenia and the World Health Organization Quality of Life Scale, respectively.

Results: The serum levels of DHEA and DHEA-S demonstrated significant gender differences, even after controlling for age effect. In the male subjects, the DHEA serum levels were positively correlated with three domains of the World Health Organization Quality of Life Scale, including physical health, social relations, and environmental dimensions. Meanwhile, the DHEA-S levels positively correlated with the performance of working memory, and pregnenolone levels had a positive correlation with working memory, verbal fluency, and Brief Assessment of Cognition in Schizophrenia composite score. However, in the female subjects, we observed a correlation only between the serum levels of DHEA-S and working memory.

Conclusion: The findings of our study indicate that neurosteroids play a vital role in cognitive function and quality of life among men but less so among women. Nevertheless, the underlying mechanisms of the gender-specific effect of neurosteroids require further investigation.

Keywords: endocrinology, DHEA, pregnenolone, cognition, quality of life

\section{Introduction}

Dehydroepiandrosterone (DHEA) is a 19-carbon steroid hormone synthesized in the inner layer (zona reticularis) of the adrenal cortex, ${ }^{1,2}$ and its sulfated form (DHEA-S) is created using DHEA sulfotransferase with reversible pathway. ${ }^{3}$ Furthermore, both DHEA and DHEA-S are synthesized in the brain, thus earning them the name "neurosteroids," and their biological characteristics include neuroprotection, neurite growth, anti-inflammatory, antioxidant, and antiglucocorticoid effects. ${ }^{5}$ DHEA(S) levels are negatively correlated to multiple domains of physical morbidities, such as ischemic heart disease, cardiovascular mortality, atherosclerosis, osteoporosis, and inflammatory diseases, as well as sexual dysfunction. ${ }^{6,7}$ Plasma DHEA(S) concentrations reach their peak levels in the third decade of life and then gradually decline ${ }^{8}$ at 
an average rate of $1 \%-4 \%$ per year in men during their $40 \mathrm{~s}$ to $80 \mathrm{~s}^{9}$ and roughly $2 \%$ per year in women. ${ }^{10}$ The aging-related decline of DHEA(S) serum levels were observed parallel to the escalating prevalence of the previously mentioned diseases. ${ }^{11}$

DHEA(S) exerts its protection mechanism by being converted into more potent sex steroids and activating androgen or estrogen receptors in tissue. ${ }^{4}$ Furthermore, DHEA has demonstrated a variety of neuroprotective and neuroexcitatory properties. ${ }^{12}$ By activating the $\sigma_{1}$ subtype receptor of NMDA receptor, DHEA(S) promotes changes of synaptic plasticity in neurons and oligodendrocytes at the prefrontal cortex, hippocampus, and striatum ${ }^{13,14}$ while performing cognitive-enhancing effects through its interaction with the $\gamma$-aminobutyric acid ${ }_{\mathrm{A}}\left(\mathrm{GABA}_{\mathrm{A}}\right)$ receptor. ${ }^{5} \operatorname{DHEA}(\mathrm{S})$ have been correlated with a number of psychiatric conditions, including depressive disorder and anxiety disorders. ${ }^{14,15}$ Higher plasma concentrations of $\operatorname{DHEA}(\mathrm{S})$ have also been associated with healthier psychological profiles, greater quality of leisure activities, ${ }^{4}$ and functional independence in men. ${ }^{16}$ Previous studies have hypothesized that frail elderly patients unsatisfied with their lives and with psychosocial stress have reduced DHEA(S) serum levels. ${ }^{7}$ A correlation between lower DHEA-S and inferior cognitive performance was also observed in both male and female seniors living in a community. ${ }^{4}$

Pregnenolone is converted from cholesterol in mitochondrion as a rate-limiting step of all neurosteroid hormones ${ }^{11}$ and can be transformed into pregnenolone sulfate upon being synthesized by pregnenolone synthase CYP/CYP450 sidechain cleavage enzyme and hydroxyl steroid sulfotransferase. ${ }^{4}$ Catalyzed by P450c17, pregnenolone is also a precursor of DHEA(S) in the brain and considered as having neurosteroid characteristics. ${ }^{7}$ Pregnenolone and its sulfate form have been declared to potentially have a neuroprotection property through their regulation of myelin synthesis by Schwann cells in both the central and peripheral nervous systems. ${ }^{1}$ Both pregnenolone and pregnenolone sulfate both bind to $\sigma_{1}$ ligands similar to DHEA(S), but pregnenolone sulfate acts as a more potent agonist than pregnenolone. ${ }^{4}$ Furthermore, pregnenolone has been associated with a number of neuropsychiatric disorders ${ }^{2,17}$ and may be associated with cognitive function and the aging process. ${ }^{2,18}$

The physiology of DHEA(S) and pregnenolone demonstrate gender differences through pubescence, adulthood, and old age..$^{9,10}$ These neuroactive steroids may exhibit genderspecific neuroprotective effects, ${ }^{19}$ and the effect of DHEA seems to benefit men more than women with regard to health maintenance. ${ }^{20}$ A prior study indicated that residential-care elderly patients with low DHEA-S levels were associated with high degrees of self-rated disability and insomnia. However, low DHEA-S levels have been linked to an increased number of pain sites only in women, not men. ${ }^{7}$

Understanding gender differences in the physiology of neurosteroids may shed light on how neurosteroids influence one's mental health and further help professionals understand the susceptibility of gender differences to neuropsychiatric diseases. However, current evidence regarding the relationships between neurosteroids, cognitive function, and quality of life have been inconclusive. ${ }^{4}$ Furthermore, most of the previous studies that have investigated gender differences in neurosteroids have targeted patients with specific diseases, ${ }^{21,22}$ and similar data among a healthy population are still lacking. Therefore, this study aimed to investigate gender differences in the relationships among neurosteroid (DHEA, DHEA-S, and pregnenolone) levels, cognitive function, and quality of life among the general population.

\section{Methods \\ Participants}

This study was approved by the Chang Gung Memorial Hospital review board. We proposed a cross-sectional design and obtained the written informed consent from all participants. The participants consisted of healthy individuals recruited from Kaohsiung Chang Gung Memorial Hospital' staff and from among community volunteers in Kaohsiung City. The recruitment criteria included the following: 1) between the ages of 18-65 years; 2) no history of illicit drug use or major psychiatric disorders (eg, psychotic disorders, bipolar disorder, major depressive disorder, dementia, or organic mental disorders) or major physical illnesses (such as genetic, endocrine, metabolic, or infectious conditions); and 3) ethnic Han Chinese origin. We ultimately enrolled a total of 47 men (mean age: 32.8 years) and 75 women (mean age: 35.4 years) in this study.

\section{Biochemistry measurements}

We collected blood samples in the morning when participants had been fasting. Blood was centrifuged immediately after it was drawn and stored at $-80^{\circ} \mathrm{C}$ until further analysis. Serum levels of DHEA were measured using a DHEA enzymelinked immunosorbent assay (ELISA) kit - ADI-900-093 Enzo Life Sciences (Farmingdale, NY, USA) (sensitivity range 12.21-50,000 pg/mL); DHEA-S levels were measured using ARCHITECT DHEA-S Reagent Kit - 8K2720 
(Wiesbaden, Germany) (sensitivity range: 0.2-961.1 $\mu \mathrm{g} / \mathrm{dL}$ ); and pregnenolone levels were measured using pregnenolone ELISA Kit - KA1912 (Jhongli, Taiwan) (sensitivity is $0.05 \mathrm{ng} / \mathrm{mL}$ ). We performed single point measurements.

\section{Cognitive function and quality of life assessments}

The cognitive functions of all participants were evaluated with the Brief Assessment of Cognition in Schizophrenia (BACS). ${ }^{23}$ BACS consists of a battery of tests that measure the aspects of cognition that are most impaired and has been strongly correlated with the real-world functioning of schizophrenic patients. ${ }^{24}$ BACS takes approximately 30 minutes to administer, generates a high completion rate in patients, and has high test-retest reliability. The tests within BACS include the List Learning Test, Digit Sequencing Task, Token Motor Task, Category Instances Test, Controlled Oral Word Association Test, Symbol Coding, and Tower of London Test, which assess verbal memory, working memory, motor speed, verbal fluency, attention, processing speed, and executive function, respectively. After all of the subtests, we calculated a composite score by comparing each patient's performance on each measure with the performance of a healthy control, which was defined as the $Z$-score or $T$-score of that sum. ${ }^{25}$ The reliability and validity of the Chinese version of the BACS have both been established in previous studies. ${ }^{26}$ The $T$-scores for each scale were used for analysis with reference to the normal population of the same age range and genders.

We evaluated quality of life with the brief version of the World Health Organization Quality of Life Scale (WHOQOL-BREF). The WHOQOL-BREF, a questionnaire self-administered by respondents, is a 26 -item version of the WHOQOL-100 assessment tool. The WHOQOL-BREF has proven useful in surveys of both general and specific populations, as well as in differentiating the health benefits produced by a wide variety of different treatments. ${ }^{27}$ This assessment is scored over four domains: Physical Capacity (seven items), Psychological Well-being (six items), Social Relationship (four items), and Environment (nine items). All items were rated on a 5-point Likert scale with a higher score indicating a higher quality of life. The WHOQOL-BREF has previously been reported to have good test-retest reliability and content validity in Taiwan. ${ }^{28}$

\section{Statistical analysis}

Data were analyzed using the Statistical Package for the Social Sciences for Windows (version 21.0; IBM Corporation, Armonk, NY, USA). Variables are presented as either mean $\pm \mathrm{SD}$ or frequency. All statistical tests are two-tailed, and differences with $P<0.05$ were considered statistically significant.

Continuous variables between male and female patients were compared using a independent $t$-test. We adopted multivariate analysis of covariance (MANCOVA) to determine intergroup differences (age, gender, and age $\times$ gender interaction) in neurosteroid levels. The MANCOVA models were also applied to estimate the potential associations between neurosteroid levels, cognitive function, and quality of life. To understand any potential gender differences, male and female subjects were separately analyzed. We set cognitive function (performance of the BACS) and quality of life (WHOQOLBREF) as the dependent variables, with DHEA, DHEA-S and pregnenolone, and cortisol as the independent variables. In order to eliminate the potential confounding effects of age and education on cognitive function, we set patients' age and educational level as covariates in the MANCOVA models. We further stratified the female participants based on the median age of 35 into a younger group ( $<35$ years old) and an older group ( $\geq 35$ years old). Pearson correlation was also adopted to estimate the correlation between neurosteroid levels, cognitive function, and quality of life.

\section{Results}

The characteristics of the 47 male subjects and 75 female subjects are shown in Table 1 . The male subjects generally had greater height $(P<0.001)$ and body weight $(P<0.001)$ than the female subjects. Besides those two factors, no significant differences in age, education level, cognitive function, or quality of life were observed between the men and women participants. Compared to the female subjects, the male subjects had higher serum levels of DHEA $(P=0.007)$, DHEA-S $(P<0.001)$, and pregnenolone $(P=0.005)$.

Figure 1 displays the relationship between age and serum levels of DHEA, DHEA-S, and pregnenolone, divided by gender. We found that DHEA $(P<0.001)$, DHEA-S $(P<0.001)$, and pregnenolone $(P=0.001)$ had a negative correlation with age. After controlling for patients' age to eliminate the inverse effect of age on neurosteroid levels, the serum levels of DHEA $(P=0.041)$ and DHEA-S $(P=0.004)$ still presented significant gender differences, but no gender difference was found with regard to pregnenolone. Furthermore, we observed no significant interactions between age and gender in the neurosteroid substrates (Table 2).

Table 3 presents the relationships between neurosteroid serum levels, cognitive function, and quality of life among the 47 male subjects, controlling for age and education level. 
Table I Comparisons of demographic data, cognitive function, quality of life, and neurosteroids levels in male and female healthy subjects

\begin{tabular}{|c|c|c|c|c|c|c|}
\hline \multirow[t]{2}{*}{ Variables } & \multicolumn{2}{|c|}{ Male $(n=47)$} & \multicolumn{2}{|c|}{ Female $(n=75)$} & \multirow{2}{*}{$\begin{array}{l}\text { Statistic } \\
\text { value }(T)\end{array}$} & \multirow[t]{2}{*}{$P$-value } \\
\hline & Mean & SD & Mean & SD & & \\
\hline \multicolumn{7}{|l|}{ Demographic data } \\
\hline Age, years & 32.83 & 11.77 & 35.35 & 10.07 & 1.258 & 0.211 \\
\hline Height, $\mathrm{cm}$ & 171.88 & 11.37 & 157.83 & 4.52 & 8.083 & $<0.00 \mathrm{I}^{\mathrm{a}}$ \\
\hline Body weight, $\mathrm{kg}$ & 75.723 & 14.76 & 55.44 & 8.35 & 8.597 & $<0.00 I^{a}$ \\
\hline Years of education & 16.02 & 2.67 & 16.23 & 2.49 & 0.431 & 0.667 \\
\hline \multicolumn{7}{|l|}{ Cognitive function (BACS) } \\
\hline Verbal memory & 52.79 & 9.76 & 50.75 & 9.67 & 1.130 & 0.261 \\
\hline Working memory & 51.06 & 9.04 & 50.43 & 6.55 & 0.450 & 0.653 \\
\hline Motor speed & 47.91 & 9.95 & 48.69 & 7.85 & 0.480 & 0.632 \\
\hline Verbal fluency & 48.96 & 10.57 & 49.91 & 9.68 & 0.509 & 0.612 \\
\hline Attention and processing speed & 49.40 & 9.35 & 51.03 & 9.73 & 0.910 & 0.365 \\
\hline Executive function & 50.00 & 9.77 & 52.79 & 7.37 & 1.789 & 0.076 \\
\hline Composite score of the BACS & 49.89 & 9.06 & 51.09 & 7.97 & 0.767 & 0.444 \\
\hline \multicolumn{7}{|l|}{ Quality of life (WHOQOL-BREF) } \\
\hline Physical health & 21.51 & 3.20 & 21.63 & 3.80 & 0.174 & 0.862 \\
\hline Psychological health & 19.47 & 3.18 & 19.60 & 3.66 & 0.204 & 0.839 \\
\hline Social relations & 13.87 & 2.46 & 14.13 & 2.72 & 0.534 & 0.594 \\
\hline Environment & 31.55 & 4.73 & 31.76 & 5.73 & 0.207 & 0.836 \\
\hline \multicolumn{7}{|l|}{ Neurosteroids } \\
\hline DHEA (ng/mL) & 9.31 & 5.08 & 7.11 & 3.76 & 2.748 & $0.007^{a}$ \\
\hline DHEA-S $(\mu g / d L)$ & 342.85 & 131.98 & 226.71 & 107.00 & 5.326 & $<0.00 I^{a}$ \\
\hline Pregnenolone $(\mathrm{ng} / \mathrm{mL})$ & 6.14 & 4.70 & 3.87 & 3.94 & 2.867 & $0.005^{\mathrm{a}}$ \\
\hline Cortisol ( $\mu g / d L)$ & 10.19 & 3.56 & 9.09 & 3.48 & 1.674 & 0.097 \\
\hline
\end{tabular}

Note: ${ }^{a} \mathrm{P}<0.05$.

Abbreviations: BACS, the Brief Assessment of Cognition in Schizophrenia; DHEA, dehydroepiandrosterone; DHEA-S, dehydroepiandrosterone-sulfate; WHOQOL-BREF, the brief version of the World Health Organization Quality of Life.

The DHEA serum levels were positively correlated to three domains of WHOQOL-BREF, which were physical health $(P=0.031)$, social relations $(P=0.047)$, and environmental dimensions $(P=0.003)$. The levels of DHEA-S were positively correlated with the performance of working memory $(P=0.031)$, while pregnenolone levels were positively correlated with working memory $(P=0.041)$, verbal fluency $(P=0.0027)$, and the composite score of the BACS $(P=0.020)$.

Table 4 lists the relationships between neurosteroid levels, cognitive function, and quality of life among the 75 female subjects, controlling for age and education level. The serum levels of DHEA-S had a positive correlation with working memory $(P=0.003)$. Otherwise, the neurosteroid levels were not correlated with other areas of cognitive function or any domain of quality of life. The age-stratified analyses (Table S1) showed the positive correlation between DHEA-S and working memory $(P=0.004)$ and a positive correlation between pregnenolone and executive function $(P=0.03)$ were only observed in the young female group ( $<35$ years old). Such correlations were not observed in the old female group ( $\geq 35$ years old) (Table S2).

\section{Discussion}

Our study's findings reveal that the correlation between neurosteroids (DHEA, DHEA-S, and pregnenolone) and cognitive function and psychological well-being may differ between the genders among a healthy population. Among men, the serum levels of DHEA were positively correlated with the domains of quality of life, and DHEA-S and pregnenolone levels were positively correlated with cognitive function. In contrast, the association between DHEA-S and working memory was the only significant finding in women. Our results indicate that neurosteroids play a vital role in the cognitive function and quality of life of males, but that role is less apparent in females.

Our findings resembled those of a previous study in which both DHEA and DHEA-S decrease through aging in both men and women, ${ }^{7,10}$ with the level dropping more steeply in men than in women..$^{10}$ Our study also demonstrated an inverse correlation between pregnenolone and age, which is comparable to previous findings..$^{9}$ A previous study demonstrated that the decrease through aging (from 20-30-year-old to 70-80-year-old) of pregnenolone was more prominent in men ( $78 \%$ decrease) than in women ( $45 \%$ decrease) ${ }^{29}$ 

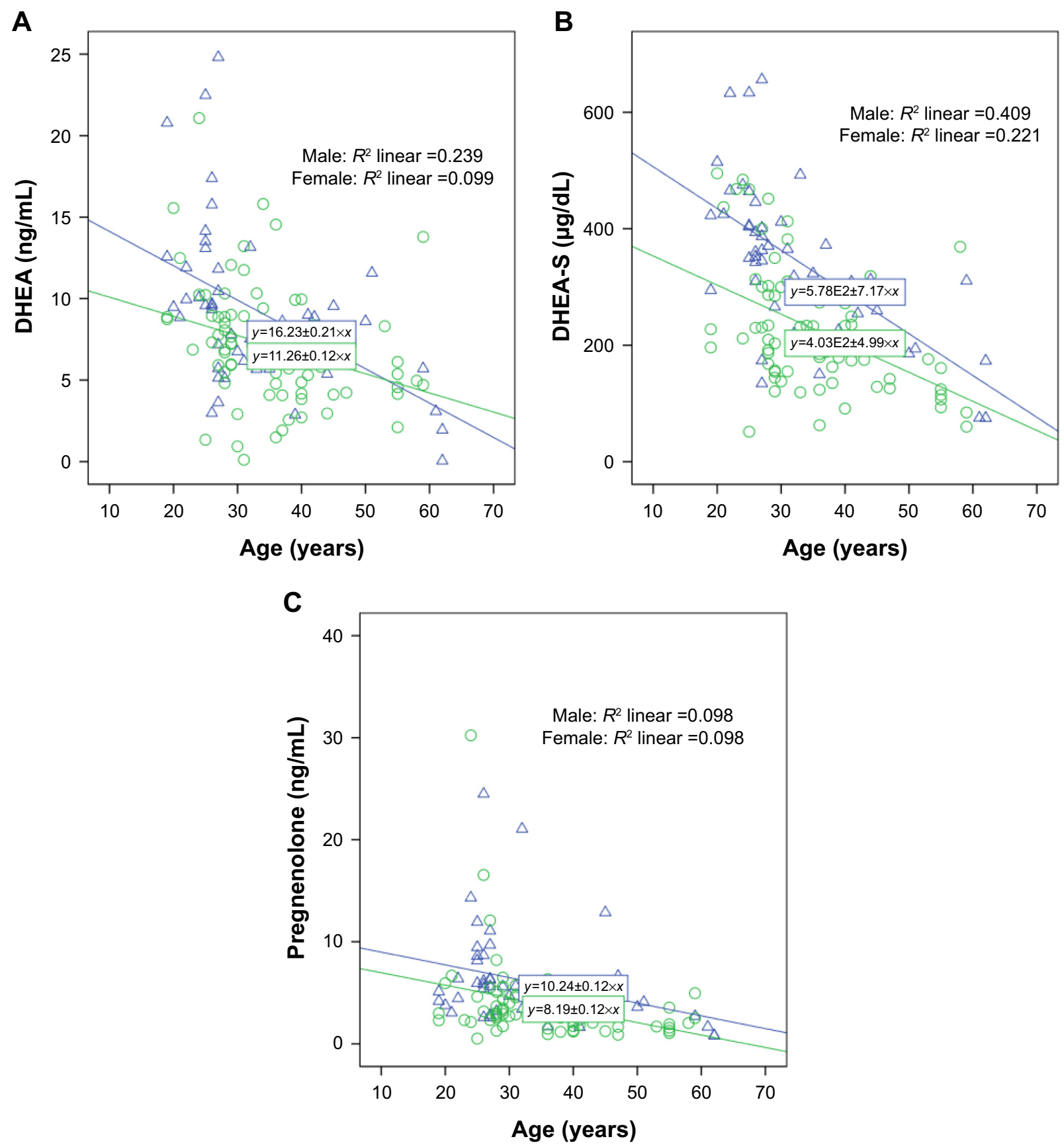

$\triangle$ Male $\circ$ Female $>$ Male $>$ Female

Figure I The relationship between age and serum levels of DHEA (A), DHEA-S (B), and pregnenolone (C), divided by gender. Abbreviations: DHEA, dehydroepiandrosterone; DHEA-S, dehydroepiandrosterone-sulfate.

Table 2 Effects of age, gender, and the interaction of age and gender on neurosteroid levels

\begin{tabular}{|c|c|c|c|c|c|c|}
\hline & \multicolumn{2}{|l|}{ DHEA } & \multicolumn{2}{|c|}{ DHEA-S } & \multicolumn{2}{|c|}{ Pregnenolone } \\
\hline & $F$ & $P$-value & $\bar{F}$ & $P$-value & $\bar{F}$ & $P$-value \\
\hline Age effect & 23.776 & $<0.00 \mathrm{I}^{\mathrm{a}}$ & 53.140 & $<0.00 \mathrm{I}^{\mathrm{a}}$ & 12.734 & $0.00 \mathrm{I}^{\mathrm{a}}$ \\
\hline Sex effect & 4.268 & $0.041^{\mathrm{a}}$ & 8.624 & $0.004^{a}$ & 0.688 & 0.409 \\
\hline Interaction of age and sex & 1.917 & 0.169 & $\mathrm{I} .703$ & 0.194 & 0.002 & 0.967 \\
\hline
\end{tabular}

Notes: Data are computed using MANCOVA. ${ }^{a} p<0.05$.

Abbreviations: DHEA, dehydroepiandrosterone; DHEA-S, dehydroepiandrosterone-sulfate; MANCOVA, multivariate analysis of covariance. 
Table 3 Correlations between neurosteroid serum levels, cognitive function, and quality of life in male subjects $(\mathrm{N}=47)$

\begin{tabular}{|c|c|c|c|c|c|c|}
\hline & \multicolumn{2}{|l|}{ DHEA } & \multicolumn{2}{|c|}{ DHEA-S } & \multicolumn{2}{|c|}{ Pregnenolone } \\
\hline & $\boldsymbol{F}$ & $P$-value & $\boldsymbol{F}$ & $P$-value & $\boldsymbol{F}$ & $P$-value \\
\hline \multicolumn{7}{|l|}{ Cognitive function (BACS) } \\
\hline Verbal memory & 0.633 & 0.431 & 0.437 & 0.512 & 1.336 & 0.255 \\
\hline Working memory & 0.278 & 0.601 & 5.034 & $0.03 I^{a}$ & 4.446 & $0.04 I^{\mathrm{a}}$ \\
\hline Motor speed & 0.012 & 0.912 & 1.331 & 0.256 & 1.387 & 0.246 \\
\hline Verbal fluency & $\mathrm{I} .878$ & 0.178 & 3.446 & 0.071 & 5.299 & $0.027^{\mathrm{a}}$ \\
\hline Attention and processing speed & 1.070 & 0.307 & 0.092 & 0.763 & 0.679 & 0.415 \\
\hline Executive function & 0.055 & 0.816 & 0.150 & 0.701 & 0.575 & 0.453 \\
\hline Composite score of the BACS & 0.014 & 0.906 & 2.527 & 0.120 & 5.887 & $0.020^{\mathrm{a}}$ \\
\hline \multicolumn{7}{|l|}{ Quality of life (WHOQOL-BREF) } \\
\hline Physical health & 5.034 & $0.03 I^{a}$ & 0.998 & 0.324 & 0.034 & 0.856 \\
\hline Psychological health & 2.028 & 0.162 & 0.193 & 0.663 & 0.179 & 0.675 \\
\hline Social relations & 4.215 & $0.047^{\mathrm{a}}$ & 0.030 & 0.862 & 2.007 & 0.164 \\
\hline Environment & 10.172 & $0.003^{a}$ & 0.256 & 0.615 & 0.814 & 0.373 \\
\hline
\end{tabular}

Notes: Data are computed using MANCOVA, controlling for age and educational level. ${ }^{a} P<0.05$.

Abbreviations: BACS, the Brief Assessment of Cognition in Schizophrenia; DHEA, dehydroepiandrosterone; DHEA-S, dehydroepiandrosterone-sulfate; MANCOVA, multivariate analysis of covariance; WHOQOL-BREF, the brief version of the World Health Organization Quality of Life.

The relationship of DHEA-S to working memory may be related to its neuroprotective property ${ }^{12}$ by promoting synaptic plasticity changes in neurons at the prefrontal cortex, hippocampus, and striatum. ${ }^{13,14}$ Evidence from fMRI has indicated that prefrontal cortex is activated during performing of working memory, ${ }^{13}$ thus suggesting the connection. Our finding of a higher circulating DHEA-S related to better working memory in both genders was consistent with previous findings. ${ }^{7,30}$ Other evidence has demonstrated the association between higher DHEA-S levels and increased association of cognitive function, even after adjusting for age and other covariates. ${ }^{4} \mathrm{~A}$ longitudinal study of older men demonstrated evidence against the correlation of DHEA-S with cognitive performance; however, the cognition testing battery did not included working memory. ${ }^{4}$ This study demonstrated the relationship between DHEA-S and working memory among the female participants and presented similar findings as another report, ${ }^{30}$ both of the studies utilized the digit sequencing task as the tool for working memory. Nevertheless, data of the report ${ }^{30}$ showed additional cognitive domains related to the DHEA-S of executive function, which may be related to a different test: executive function, which was examined by the Tower of London test in our study compared to Trail Making Test B in the other study. One study of 394 community-dwelling women over the age of 65 years old showed no relationship between DHEA-S concentrations and cognitive function or changes in cognitive performance over time. ${ }^{7}$ Our age-stratified analyses also showed that the

Table 4 Correlations between neurosteroid serum levels, cognitive function, and quality of life in female subjects ( $N=75)$

\begin{tabular}{|c|c|c|c|c|c|c|}
\hline & \multicolumn{2}{|c|}{ DHEA } & \multicolumn{2}{|c|}{ DHEA-S } & \multicolumn{2}{|c|}{ Pregnenolone } \\
\hline & $\boldsymbol{F}$ & $P$-value & $\boldsymbol{F}$ & $P$-value & $\boldsymbol{F}$ & $P$-value \\
\hline \multicolumn{7}{|l|}{ Cognitive function (BACS) } \\
\hline Verbal memory & 0.920 & 0.341 & 0.772 & 0.383 & 0.002 & 0.964 \\
\hline Working memory & 0.942 & 0.335 & 9.245 & $0.003^{\mathrm{a}}$ & 1.029 & 0.314 \\
\hline Motor speed & 0.028 & 0.868 & 0.041 & 0.840 & 0.011 & 0.918 \\
\hline Verbal fluency & 2.655 & 0.108 & 0.913 & 0.343 & 1.254 & 0.267 \\
\hline Attention and processing speed & 1.835 & 0.180 & 0.117 & 0.733 & 0.537 & 0.466 \\
\hline Executive function & 1.305 & 0.257 & 1.794 & 0.185 & 2.989 & 0.088 \\
\hline Composite score of the BACS & 1.027 & 0.314 & 3.833 & 0.054 & 1.055 & 0.308 \\
\hline \multicolumn{7}{|l|}{ Quality of life (WHOQOL-BREF) } \\
\hline Physical health & 0.287 & 0.594 & 0.002 & 0.965 & 0.324 & 0.571 \\
\hline Psychological health & 0.000 & 0.999 & 1.186 & 0.280 & 0.003 & 0.956 \\
\hline Social relations & 0.599 & 0.442 & 0.392 & 0.534 & 0.016 & 0.900 \\
\hline Environment & 0.000 & 0.992 & 0.002 & 0.966 & 0.001 & 0.971 \\
\hline
\end{tabular}

Notes: Data are computed using MANCOVA, controlling for age and educational level. ${ }^{a} P<0.05$.

Abbreviations: BACS, the Brief Assessment of Cognition in Schizophrenia; DHEA, dehydroepiandrosterone; DHEA-S, dehydroepiandrosterone-sulfate; MANCOVA, multivariate analysis of covariance; WHOQOL-BREF, the brief version of the World Health Organization Quality of Life. 
positive correlation between DHEA-S and working memory only exhibited in the young female group, but not in old female group. It implies that DHEA-S may exert a divergent effect on cognition among various age groups.

In our research, pregnenolone was significantly correlated with the working memory, verbal fluency, and overall presentation of cognitive function in healthy men. However, in females, the relationship between pregnenolone serum concentration and executive function was found in younger group ( $<35$ years old). While some studies have reported the biological effects of pregnenolone per se due to its poor activity on the traditional neurosteroid targets, ${ }^{2}$ its sulfate ester is more potentially affective on various mechanisms. Pregnenolone and its sulfate ester not only act as positive modulators on NMDA receptors ${ }^{2,7}$ with the effects of calcium ion influx and consequently the synthesis of pregnenolone and pregnenolone sulfate in the hippocampus, ${ }^{31}$ but also enhance inwardly rectifying potassium- 2.3 channels, which are abundantly expressed in the forebrain area related to cognition, memory, and emotion. ${ }^{6}$ Furthermore, other animal studies revealed modulation by pregnenolone sulfate of acetylcholine neurotransmission that prominently affects cognitive processes. ${ }^{3}$ The affirmative effects of pregnenolone on working memory ${ }^{7}$ and synaptic plasticity in memoryrelated brain areas ${ }^{32}$ were also reported in animal studies. This study's results suggested that in healthy males, pregnenolone or perhaps its sulfate ester correlates with cognitive function per se and not through the effect of its downstream metabolites (eg, DHEA, DHEA-S). However, little evidence has been found to support the correlation between pregnenolone and cognitive function in humans; prospective studies are necessary to illustrate the underlying mechanism of this relationship.

As for quality of life, DHEA is significantly consistent with physical health, social relations, and environmental factors in the quality of life of healthy men in our findings. DHEA has been proven to have a potential neuroprotective ability against excitatory amino acid-induced neurotoxicity both in vivo and in vitro. ${ }^{4}$ However, DHEA has a more protective effect on men's health, ${ }^{33}$ cardiovascular outcomes, ${ }^{16}$ and bone protection through its conversion to androgen and estrogen and regulation of osteogenesis activity, ${ }^{8}$ as well as better performance against stressors in a military setting, ${ }^{34}$ ability to perform daily activities, ${ }^{10}$ higher ADL,${ }^{21}$ and lower susceptibility to mood disturbances. ${ }^{34}$ The gender differences in this study may result from such health-related behaviors as smoking or drinking or cardiovascular risk factors; however, some evidence has refuted the differences to support these hypotheses..$^{33}$ Our data showed that the quality of life among the female participant group did not correlate to either of the neurosteroids, differing from the male group's findings. The relationship of endogenous DHEA-S to the healthier life of men but not women has been observed in both Western and non-Western populations. ${ }^{33}$ While most studies focusing on women showed no significant effects on quality of life, the DHEA concentration also did not affect the mortality in disabled women. ${ }^{33}$ Recent studies have demonstrated a relationship between executive functioning, working memory, vocabulary measures, and both the physical and psychological health domains of the WHOQOL-BREF in healthy older adults, ${ }^{35}$ suggesting that other neuropsychiatric systems, such as estrogen, that are related to cognition and quality of life in the female group. ${ }^{36}$

Several biological effects may account for the gender difference in the correlation between neurosteroids and cognition. In the early postmenopausal period, estrogen has also been claimed to have an affirmative influence on the prefrontal cortex and hippocampus. ${ }^{37}$ Estradiol was related to the maintenance of various cognitive domains, including attention and verbal and visual working memory, ${ }^{21,37}$ while estrogen receptors are present in such brain areas as the cerebral cortex, hypothalamus, and the pyramidal cells CA 1 in the ventral hippocampus, which is vital for memory. ${ }^{21}$ Estrogen was found to increase dendritic spine density in the CA 1 neurons in the hippocampus of female rats, ${ }^{21}$ as well as to stimulate CAMP and MAPK pathways and C protein on the calcium channel for neuroprotective properties. ${ }^{38}$ Estrogen has also been reported to have a cofactor effect on the metabolism of acetylcholine, ${ }^{39}$ a neurotransmitter that plays a vital role in attention, learning, and memory functions. ${ }^{21}$ Other recent reports have demonstrated estrogen-modulated cholinergic functioning through estrogen receptors GPR30 in the basal forebrain..$^{40}$ In vitro studies have implicated estrogen in the degradation of $\beta$-amyloid precursor protein from its accumulation into $\beta$-amyloid through astrocyte activation. ${ }^{21,41}$ Estrogen has an increasing modulation effect on the synthesis of dopamine, ${ }^{42}$ a neurotransmitter related to attention, processing speed, working memory, and positive affect. ${ }^{42}$ While estrogen exhibits a positive effect on serotonin ${ }^{38}$ and the binding of serotonin to $5-\mathrm{HT}_{2 \mathrm{~A}}$ receptor at the prefrontal cortex, which is related to verbal working memory in postmenopausal women, ${ }^{43}$ other neuroprotective mechanisms of estrogen were noted, such as increased BDNF and CREB expression. ${ }^{44}$

Female hippocampal neurons produce estradiol in order to uphold long-term potential memory; however, 
this condition was not observed in males in animal studies. ${ }^{45}$ The female hippocampus has a higher amount of estradiol than that of men, ${ }^{46}$ with a correlation with both volume and cognitive function. ${ }^{36}$ Although the serum concentration of estradiol was positively related to the hippocampal volume in men, the relationship revealed no consequences on memory performance, ${ }^{47}$ while the inhibition of estrogen was also not related to cognitive function in prepubescent males. ${ }^{48}$ The reinforcing effect of testosterone on working memory outstood estrogen in male rats, while the effect on visual working memory was contrary, ${ }^{49}$ thus supporting the gender differences of neurosteroids' physiology on cognitive function.

A systemic review enrolled 23 randomized controlled trials, and 1,188 women indicated no association of DHEA use with improvement in sexual function, ${ }^{4}$ and the DHEA supplement showed no benefit with regard to improving the quality of life in either peri- or postmenopausal women ${ }^{27}$ or profit on cognitive performance or well-being in healthy elderly subjects. ${ }^{10}$ Another hypothesis suggests that the Taiwanese diet, which contains soy-based nutrition, has a higher level of genistein than those in Western society, which may lower the effects of DHEA-derived estrogen. ${ }^{16}$ Overall, no conclusions have been reached regarding the advantage of DHEA supplementation for improving well-being in healthy older adults; ${ }^{4,10}$ both the affirmative stance and negative point of view ${ }^{10,45}$ have been postulated, indicating the lack of standard evaluating measures for quality of life. DHEA(S) seemed to serve more as a predictor than a therapeutic index, but more prospective studies are still needed to support the hypothesis.

This study has several limitations. First, this was a crosssectional study with rather small sample size. While we found an association between neurosteroids and cognitive function in men, the causality and underlying mechanism remain unclear. Moreover, the study statistical analyses were performed in an exploratory manner. Therefore, a Bonferroni correction for multiple comparisons was not performed, and the possibility of false-positive findings might not be excluded. In addition, the serum levels of neurosteroids are detected by ELISA in this study, but mass spectrometry may provide more accurate analysis. Second, several important factors that may simultaneously influence neurosteroid levels and mental health, such as life stress, lifestyle (smoking, alcohol consumption, diet, and exercise), physical comorbidities (surgical history of gynecology which may cause disturbance of variety of hormones), and medication as contraceptive therapy or other medication affecting neurosteroids, were not identified in this study. ${ }^{21,39}$ Third, participants recruited in this study were within a wide age range (18-65 years), and the potential effect of neurosteroids on cognition and quality of life might be varied in different age groups. Among the female participants, the study did not address the influence of menstruation status between the covariate, even though DHEA(S) levels and their effect on cognition are known to differ between pre- and postmenopausal status. ${ }^{4}$ Lastly, relative to serum neurosteroids levels, the levels in cerebrospinal fluid may closely reflect actual physiological situations in brain. ${ }^{50}$ Therefore, the serum samples used for measuring the neurosteroid levels in this study may not necessarily represent the concentration and activity of neurosteroids in the brain..$^{50}$

\section{Conclusion}

The findings of our study demonstrate that the correlation between neurosteroids (DHEA, DHEA-S, and pregnenolone) and cognitive function and psychological well-being may differ with gender. We thus hypothesize that neurosteroids play a vital role in cognitive function and quality of life among men, but less so among women. Nevertheless, the underlying mechanisms of the gender-specific effect on cognition and quality of life warrant further clarification through future studies.

\section{Acknowledgment}

This study was supported by the grant from Chang Gung Memorial Hospital, Taiwan (CMRPG8C1051 and CMRPG8C1291). The authors wish to express their deepest gratitude to all the patients who participated in this study.

\section{Author contributions}

All authors contributed to data analysis, drafting and revising the article, gave final approval of the version to be published, and agree to be accountable for all aspects of the work.

\section{Disclosure}

The authors report no conflicts of interest in this work.

\section{References}

1. Zhu TS, Glaser M. Regulatory role of cytochrome P450scc and pregnenolone in myelination by rat Schwann cells. Mol Cell Biochem. 2008; 313(1-2):79-89.

2. Vallée M. Neurosteroids and potential therapeutics: focus on pregnenolone. J Steroid Biochem Mol Biol. 2016;160:78-87.

3. Mayo W, George O, Darbra S, et al. Individual differences in cognitive aging: implication of pregnenolone sulfate. Prog Neurobiol. 2003;71(1): 43-48.

4. Maninger N, Wolkowitz OM, Reus VI, Epel ES, Mellon SH. Neurobiological and neuropsychiatric effects of dehydroepiandrosterone (DHEA) and DHEA sulfate (DHEAS). Front Neuroendocrinol. 2009; 30(1):65-91. 
5. Stárka L, Dušková M, Hill M. Dehydroepiandrosterone: a neuroactive steroid. J Steroid Biochem Mol Biol. 2015;145:254-260.

6. Inanobe A, Fujita A, Ito M, Tomoike H, Inageda K, Kurachi Y. Inward rectifier $\mathrm{K}+$ channel Kir2.3 is localized at the postsynaptic membrane of excitatory synapses. Am J Physiol Cell Physiol. 2002;282(6):C1396-C1403.

7. Racchi M, Balduzzi C, Corsini E. Dehydroepiandrosterone (DHEA) and the aging brain: flipping a coin in the "fountain of youth". CNS Drug Rev. 2003;9(1):21-40.

8. Inoue S. Molecular mechanism involved in estrogen action and its role in bone metabolism and brain function. Nihon Naika Gakkai Zasshi. 2003;92(9):1684-1689.

9. Hill M, Lukác D, Lapcík O, et al. Age relationships and sex differences in serum levels of pregnenolone and 17-hydroxypregnenolone in healthy subjects. Clin Chem Lab Med. 1999;37(4):439-447.

10. Kritz-Silverstein D, von Mühlen D, Laughlin GA, Bettencourt R. Effects of dehydroepiandrosterone supplementation on cognitive function and quality of life: the DHEA and Well-Ness (DAWN) Trial. $J$ Am Geriatr Soc. 2008;56(7):1292-1298.

11. Auchus RJ. Overview of dehydroepiandrosterone biosynthesis. Semin Reprod Med. 2004;22(4):281-288.

12. Taylor MK. Dehydroepiandrosterone and dehydroepiandrosterone sulfate: anabolic, neuroprotective, and neuroexcitatory properties in military men. Mil Med. 2013;178(1):100-106.

13. Na DG, Ryu JW, Byun HS, et al. Functional MR imaging of working memory in the human brain. Korean J Radiol. 2000;1(1):19-24.

14. Hogervorst E. Estrogen and the brain: does estrogen treatment improve cognitive function? Menopause Int. 2013;19(1):6-19.

15. Oulis P, Masdrakis VG, Markianos M. Testosterone and dehydroepiandrosterone sulfate in female anxious and non-anxious major depression. Int J Psychiatry Clin Pract. 2014;18(1):21-24.

16. Glei DA, Goldman N, Weinstein M, Liu IW. Dehydroepiandrosterone sulfate (DHEAS) and health: does the relationship differ by sex? Exp Gerontol. 2004;39(3):321-331.

17. Daftary S, Yon JM, Choi EK, et al. Microtubule associated protein 2 in bipolar depression: impact of pregnenolone. J Affect Disord. 2017; 218:49-52.

18. Mayo W, Le Moal M, Abrous DN. Pregnenolone sulfate and aging of cognitive functions: behavioral, neurochemical, and morphological investigations. Horm Behav. 2001;40(2):215-217.

19. Melcangi RC, Giatti S, Garcia-Segura LM. Levels and actions of neuroactive steroids in the nervous system under physiological and pathological conditions: Sex-specific features. Neurosci Biobehav Rev. 2016;67:25-40.

20. Birkenhäger-Gillesse EG, Derksen J, Lagaay AM. Dehydroepiandrosterone sulphate (DHEAS) in the oldest old, aged 85 and over. Ann NY Acad Sci. 1994;719:543-552.

21. Sherwin BB, Henry JF. Brain aging modulates the neuroprotective effects of estrogen on selective aspects of cognition in women: a critical review. Front Neuroendocrinol. 2008;29(1):88-113.

22. Heald AH, Walther A, Davis JRE, et al. No Difference in Mood and Quality of Life in DHEA-S Deficient Adults with Addison's Disease vs. Type 2 Diabetes Patients with Normal DHEA-S Levels: Implications for Management of These Conditions. Front Psychol. 2017;8:764.

23. Keefe RS, Goldberg TE, Harvey PD, Gold JM, Poe MP, Coughenour L. The Brief Assessment of Cognition in Schizophrenia: reliability, sensitivity, and comparison with a standard neurocognitive battery. Schizophr Res. 2004;68(2-3):283-297.

24. Keefe RS, Poe M, Walker TM, Kang JW, Harvey PD. The Schizophrenia Cognition Rating Scale: an interview-based assessment and its relationship to cognition, real-world functioning, and functional capacity. Am J Psychiatry. 2006;163(3):426-432.

25. Keefe RS, Harvey PD, Goldberg TE, et al. Norms and standardization of the Brief Assessment of Cognition in Schizophrenia (BACS). Schizophr Res. 2008;102(1-3):108-115.

26. Wang LJ, Lin PY, Lee Y, et al. Validation of the Chinese version of Brief Assessment of Cognition in Schizophrenia. Neuropsychiatr Dis Treat. 2016;12:2819-2826.
27. Development of the World Health Organization WHOQOL-BREF quality of life assessment. The WHOQOL Group. Psychol Med. 1998; 28(3):551-558.

28. Yao G, Chung CW, Yu CF, Wang JD. Development and verification of validity and reliability of the WHOQOL-BREF Taiwan version. J Formos Med Assoc. 2002;101(5):342-351.

29. Labrie F, Bélanger A, Cusan L, Gomez JL, Candas B. Marked decline in serum concentrations of adrenal C19 sex steroid precursors and conjugated androgen metabolites during aging. J Clin Endocrinol Metab. 1997;82(8):2396-2402.

30. Davis SR, Shah SM, McKenzie DP, Kulkarni J, Davison SL, Bell RJ. Dehydroepiandrosterone sulfate levels are associated with more favorable cognitive function in women. J Clin Endocrinol Metab. 2008;93(3): 801-808.

31. Kimoto T, Tsurugizawa T, Ohta Y, et al. Neurosteroid synthesis by cytochrome p450-containing systems localized in the rat brain hippocampal neurons: N-methyl-D-aspartate and calcium-dependent synthesis. Endocrinology. 2001;142(8):3578-3589.

32. $\mathrm{Bu} \mathrm{J}, \mathrm{Zu} \mathrm{H}$. Effects of pregnenolone intervention on the cholinergic system and synaptic protein 1 in aged rats. Int J Neurosci. 2014;124(2): $117-124$.

33. Goldman N, Glei DA. Sex differences in the relationship between DHEAS and health. Exp Gerontol. 2007;42(10):979-987.

34. Wemm S, Koone T, Blough ER, Mewaldt S, Bardi M. The role of DHEA in relation to problem solving and academic performance. Biol Psychol. 2010;85(1):53-61.

35. Constantinidou F, Prokopiou J, Nikou M, Papacostas S. CognitiveLinguistic Performance and Quality of Life in Healthy Aging. Folia Phoniatr Logop. 2015;67(3):145-155

36. Protopopescu X, Butler T, Pan H, et al. Hippocampal structural changes across the menstrual cycle. Hippocampus. 2008;18(10):985-988.

37. Leblanc ES, Janowsky J, Chan BK, Nelson HD. Hormone replacement therapy and cognition: systematic review and meta-analysis. JAMA. 2001;285(11):1489-1499.

38. Epperson CN, Amin Z, Ruparel K, Gur R, Loughead J. Interactive effects of estrogen and serotonin on brain activation during working memory and affective processing in menopausal women. Psychoneuroendocrinology. 2012;37(3):372-382.

39. Craig LA, Hong NS, McDonald RJ. Revisiting the cholinergic hypothesis in the development of Alzheimer's disease. Neurosci Biobehav Rev. 2011;35(6):1397-1409.

40. Gibbs RB, Nelson D, Hammond R. Role of GPR30 in mediating estradiol effects on acetylcholine release in the hippocampus. Horm Behav. 2014;66(2):339-345.

41. Vincent B, Smith JD. Effect of estradiol on neuronal Swedish-mutated beta-amyloid precursor protein metabolism: reversal by astrocytic cells. Biochem Biophys Res Commun. 2000;271(1):82-85.

42. Riccardi P, Park S, Anderson S, et al. Sex differences in the relationship of regional dopamine release to affect and cognitive function in striatal and extrastriatal regions using positron emission tomography and $\left[{ }^{18} \mathrm{~F}\right]$ fallypride. Synapse. 2011;65(2):99-102.

43. Kugaya A, Epperson CN, Zoghbi S, et al. Increase in prefrontal cortex serotonin $2 \mathrm{~A}$ receptors following estrogen treatment in postmenopausal women. Am J Psychiatry. 2003;160(8):1522-1524.

44. Zhou J, Zhang H, Cohen RS, Pandey SC. Effects of estrogen treatment on expression of brain-derived neurotrophic factor and cAMP response element-binding protein expression and phosphorylation in rat amygdaloid and hippocampal structures. Neuroendocrinology. 2005;81(5):294-310.

45. Vierk R, Brandt N, Rune GM. Hippocampal estradiol synthesis and its significance for hippocampal synaptic stability in male and female animals. Neuroscience. 2014;274:24-32.

46. Fester L, Rune GM. Sexual neurosteroids and synaptic plasticity in the hippocampus. Brain Res. 2015;1621:162-169.

47. Vierk R, Bayer J, Freitag S, et al. Structure-function-behavior relationship in estrogen-induced synaptic plasticity. Horm Behav. 2015;74: 139-148. 
48. Hero M, Maury S, Luotoniemi E, Service E, Dunkel L. Cognitive effects of aromatase inhibitor therapy in peripubertal boys. Eur J Endocrinol. 2010;163(1):149-155.

49. Gibbs RB. Testosterone and estradiol produce different effects on cognitive performance in male rats. Horm Behav. 2005;48(3):268-277.
50. Kancheva R, Hill M, Novák Z, Chrastina J, Kancheva L, Stárka L. Neuroactive steroids in periphery and cerebrospinal fluid. Neuroscience. 2011;191:22-27. 


\section{Supplementary materials}

Table SI Correlations between neurosteroid serum levels, cognitive function, and quality of life in older female subjects ( $<35$ years old, $\mathrm{N}=40$ )

\begin{tabular}{|c|c|c|c|c|c|c|}
\hline & \multicolumn{2}{|l|}{ DHEA } & \multicolumn{2}{|c|}{ DHEA-S } & \multicolumn{2}{|c|}{ Pregnenolone } \\
\hline & $r$ & $P$-value & $r$ & $P$-value & $r$ & $P$-value \\
\hline \multicolumn{7}{|l|}{ Cognitive function (BACS) } \\
\hline Verbal memory & 0.02 & 0.904 & 0.166 & 0.307 & 0.086 & 0.599 \\
\hline Working memory & 0.175 & $0.28 \mathrm{I}$ & 0.448 & $0.004^{a}$ & -0.008 & 0.959 \\
\hline Motor speed & -0.057 & 0.726 & 0.074 & 0.648 & -0.074 & 0.652 \\
\hline Verbal fluency & -0.248 & 0.122 & 0.151 & 0.352 & 0.184 & 0.257 \\
\hline Attention and processing speed & -0.134 & $0.4 I I$ & -0.01 & 0.949 & 0.172 & 0.288 \\
\hline Executive function & -0.123 & 0.451 & 0.092 & 0.574 & 0.344 & $0.03^{\mathrm{a}}$ \\
\hline Composite score of the BACS & -0.059 & 0.719 & 0.273 & 0.088 & 0.219 & 0.175 \\
\hline \multicolumn{7}{|l|}{ Quality of life (WHOQOL-BREF) } \\
\hline Physical health & -0.018 & 0.914 & -0.065 & 0.691 & 0.044 & 0.786 \\
\hline Psychological health & 0.1 & 0.537 & 0.172 & 0.289 & -0.007 & 0.967 \\
\hline Social relations & -0.003 & 0.985 & 0.032 & 0.842 & 0.047 & 0.774 \\
\hline Environment & -0.022 & 0.893 & -0.112 & 0.493 & -0.157 & 0.334 \\
\hline
\end{tabular}

Notes: Data are computed using Pearson Correlation. ${ }^{a} \mathrm{P}<0.01$.

Abbreviations: BACS, the Brief Assessment of Cognition in Schizophrenia; DHEA, dehydroepiandrosterone; DHEA-S, dehydroepiandrosterone-sulfate; WHOQOL-BREF, the brief version of the World Health Organization Quality of Life.

Table S2 Correlations between neurosteroid serum levels, cognitive function, and quality of life in older female subjects ( $\geq 35$ years old, $\mathrm{N}=35$ )

\begin{tabular}{|c|c|c|c|c|c|c|}
\hline & \multicolumn{2}{|l|}{ DHEA } & \multicolumn{2}{|c|}{ DHEA-S } & \multicolumn{2}{|c|}{ Pregnenolone } \\
\hline & $r$ & $P$-value & $r$ & $P$-value & $r$ & $P$-value \\
\hline \multicolumn{7}{|l|}{ Cognitive function (BACS) } \\
\hline Verbal memory & -0.233 & 0.178 & 0.022 & 0.902 & -0.075 & 0.67 \\
\hline Working memory & 0.092 & 0.598 & 0.208 & 0.231 & -0.018 & 0.917 \\
\hline Motor speed & 0.014 & 0.938 & -0.072 & 0.683 & 0.244 & 0.158 \\
\hline Verbal fluency & -0.014 & 0.937 & 0.183 & 0.292 & 0.196 & 0.259 \\
\hline Attention and processing speed & -0.191 & 0.271 & 0.032 & 0.853 & 0.032 & 0.856 \\
\hline Executive function & -0.067 & 0.7 & 0.273 & 0.112 & 0.034 & 0.845 \\
\hline Composite score of the BACS & -0.08 & 0.648 & 0.184 & 0.291 & 0.159 & 0.361 \\
\hline \multicolumn{7}{|l|}{ Quality of life (WHOQOL-BREF) } \\
\hline Physical health & -0.157 & 0.368 & 0.001 & 0.996 & -0.045 & 0.796 \\
\hline Psychological health & -0.028 & 0.875 & 0.037 & 0.834 & 0.075 & 0.668 \\
\hline Social relations & $-0.14 \mid$ & 0.42 & 0.084 & 0.63 & -0.028 & 0.872 \\
\hline Environment & -0.061 & 0.729 & -0.009 & 0.959 & -0.062 & 0.723 \\
\hline
\end{tabular}

Note: Data are computed using Pearson Correlation.

Abbreviations: BACS, the Brief Assessment of Cognition in Schizophrenia; DHEA, dehydroepiandrosterone; DHEA-S, dehydroepiandrosterone-sulfate; WHOQOL-BREF, the brief version of the World Health Organization Quality of Life.

Neuropsychiatric Disease and Treatment

\section{Publish your work in this journal}

Neuropsychiatric Disease and Treatment is an international, peerreviewed journal of clinical therapeutics and pharmacology focusing on concise rapid reporting of clinical or pre-clinical studies on a range of neuropsychiatric and neurological disorders. This journal is indexed on PubMed Central, the 'PsycINFO' database and CAS,

\section{Dovepress}

and is the official journal of The International Neuropsychiatric Association (INA). The manuscript management system is completely online and includes a very quick and fair peer-review system, which is all easy to use. Visit http://www.dovepress.com/testimonials.php to read real quotes from published authors. 